\title{
La Chine dans l'espace cardinal
}

In: Revue française de science politique, 36 e année, ${ }^{\circ} 6$, 1986. pp. 787-809.

Citer ce document / Cite this document :

Domenach Jean-Luc. La Chine dans l'espace cardinal. In: Revue française de science politique, $36 \mathrm{e}$ année, $\mathrm{n}^{\circ} 6,1986 . \mathrm{pp} .787-$ 809.

http://www.persee.fr/web/revues/home/prescript/article/rfsp_0035-2950_1986_num_36_6_394278 


\section{LA CHINE DANS L'ESPACE CARDINAL}

JEAN-LUC DOMENACH

$\mathbf{N}$ ÉE d'une interrogation sur la contrainte et l'autonomie dans l'espace international contemporain, la problématique des relations cardinales s'inscrit dans la lignée intellectuelle de tous ceux historiens ou politistes - pour qui les rapports entre l'interne et l'externe ne sont, ni statiques, ni univalents. Elle se développe suivant deux intuitions. La première est que l'espace international contemporain est polarisé par des points cardinaux qui sont à la fois position et attraction, sens et puissance, contrainte et recours. La seconde est que les acteurs politiques du Sud, dans le même temps où ils sont façonnés par l'espace qui les immerge, recourent à ces pôles pour se déterminer et pour modifier leur environnement ${ }^{1}$.

\section{LA FORMATION DE L'ESPACE CARDINAL}

Pour préciser l'apport de l'expérience historique chinoise à la problématique des relations cardinales, une première distinction s'impose entre les espaces cardinaux pré-moderne et moderne. Car les Empires chinois se représentaient un univers défini par quatre points cardinaux. De cet univers, ils occupaient le milieu, qui seul était éclairé par la civilisation. Peu importe que dans les faits la Chine n'ait jamais été un isolat. L'essentiel est que la force et la vitalité de son ordre interne lui donnaient les moyens de se penser comme le milieu du monde et de récuser les valeurs de l'étranger.

L'histoire moderne chinoise commence donc quand, de façon brutale, le monde extérieur s'impose à l'Empire chinois. Classiquement, cette histoire est celle d'un asservissement, puis d'une émancipation nationaliste. Mais elle est à la fois déterminée et éclairée par la formation de l'espace cardinal dans lequel la Chine se meut depuis. Cet espace est prégnant : la Chine ne peut s'y mouvoir qu'à la condition de reconnaître qu'elle en est pénétrée de toute part. Il n'est pas indifférencié, mais polarisé par des forces qui sont à la fois des menaces et des ressources.

De cet espace cardinal, le pôle occidental se constitue le premier. De l'Ouest géographique arrivent des puissances qui, par leur supériorité militaire, révèlent l'inadéquation dramatique du vieil Empire aux normes de la modernité ${ }^{2}$. Dans un premier temps, cet Ouest paraît résumer le

1. Laïdi (Zaki), sous la direction de, L'URSS vue du Tiers Monde, Paris, Karthala, 1984 ; Domenach (Jean-Luc), Laïdi (Zaki), «Les relations cardinales, réflexions sur le marché idéologique international \#, Esprit, novembre 1985, p. 55-64.

2. Skocpol (Theda), Etats et révolutions sociales; la révolution en France, en Russie et en Chine, traduction Noëlle Burgi, Paris, Fayard, 1985. 
Nord industriel. Sa surpuissance renvoie une Chine autrefois centrale aux marges méridionales du monde - zone indifférenciée que le Nord/Ouest prétend illuminer et que ses acteurs découpent; la guerre de 1914-1918 n'est que faussement mondiale - par l'attraction dans le vrai monde des tranchées de la chair à canon « indigène ", avec son contingent de coolies annamites et chinois importés par cargos entiers. De cette guerre sort cependant, en 1917, le dernier pôle de l'espace cardinal. A l'intérieur du Nord géographique et industriel naît, après la Révolution d'octobre, un anti-Ouest qui apparaît comme un Est idéologique et politique. Pour les nationalistes chinois de tout bord, la formation de l'Union Soviétique est un événement extraordinaire parce qu'elle s'inscrit dans le monde supérieur tout en désignant une voie nouvelle pour lui résister.

Désormais, l'espace-monde cesse d'être seulement pour la Chine celui d'une contrainte indépassable, il devient un champ d'options. Dès lors que ce champ se diversifie, que donc apparaît avec le choix l'espoir de l'émancipation, les nationalistes admettent la nécessité d'emprunter au Nord. Mais ils se divisent sur la nature et sur l'origine de l'emprunt. Les uns n'entendent emprunter que des techniques, les autres des modèles complets, c'est-à-dire les ensembles de normes culturelles, idéologiques et politiques qui ont, selon eux, engendré la supériorité de la puissance nordique. Le débat oppose ensuite, et de plus en plus, les partisans de l'Ouest et ceux de l'Est. Jusqu'alors investie par le Nord, la Chine peut désormais s'efforcer de se réapproprier ses normes concurrentes : la mondialisation du grand conflit idéologique ${ }^{1}$ ouvre dans le Sud des espaces d'autonomie.

Les nationalistes les plus radicaux choisissent d'emprunter le modèle soviétique. En effet, l'URSS apparaît comme l'anti-Occident du Nord; son modèle a prouvé son efficacité en permettant au "pouvoir rouge " de résister à l'encerclement des "puissances "; c'est donc autant un modèle de construction du pouvoir qu'un exemple social ; il comprend une volonté et des techniques d'exportation. Surtout, il s'adapte aisément aux besoins de nationalistes qui ont choisi la voie de la révolution ${ }^{2}$. Car c'est tout d'abord une explication commode - au travers des concepts d'impérialisme et de capitalisme - des maux que la Chine subit depuis quelques décennies. C'est en second lieu une légitimation eschatologique de la voie révolutionnaire. C'est enfin un ensemble de techniques organisationnelles - le parti du prolétariat, le centralisme démocratique, la dictature du prolétariat, le front uni...

Que le communisme l'ait emporté en Chine malgré puis sans le soutien soviétique est une donnée de fait connue qu'expliquent de nombreux facteurs ${ }^{3}$. Le point important, pour nous, est que la validité opérationnelle du modèle soviétique figure parmi ces facteurs. L'indépendance pratique du PCC n'a pas limité mais au contraire permis de

1. Ansart (Pierre), Idéologies, conflits et pouvoir, Paris, PUF, 1977, p. 259.

2. Chevrier (Yves), «Mort et transfiguration: le modèle russe dans la révolution chinoise ", Extrême Orient-Extrême Occident, 2, 1983, p. 41-108.

3. Bianco (Lucien), Les origines de la révolution chinoise, Paris, Fayard, 1967. 
réaliser l'instrumentalisation de ce modèle. La soviétisation du PCC a été d'autant plus intense qu'elle provenait d'une conviction. Le communisme chinois s'est soviétisé de lui-même, jusqu'à la caricature politique ou sémantique : il y a eu des purges de «trotskystes" dans des bases rouges reculées, et le vocabulaire stalinien a véritablement structuré la langue de bois naissante. En même temps, l'éloignement physique facilitait les manipulations nécessitées par les dorénavant fameuses "conditions spécifiques " de la Chine, et d'abord la double substitution, opérée sous l'impulsion de Mao, de la paysannerie au prolétariat et de la guérilla à la lutte urbaine. Mais l'instrumentalisation du modèle soviétique, malgré sa cohérence apparente, a placé la révolution communiste en Chine sous le signe d'une ambivalence instable dont elle ne s'est par la suite jamais évadée : nationalisme et emprunt à l'étranger, acceptation et refus de la contrainte extérieure, emprunt de techniques ou du modèle. La contradiction n'était pas durablement dépassée, mais temporairement anesthésiée par une combinaison de nature stratégique.

\section{LA ClÔTURE TOTALITAIRE}

Le paradoxe du communisme chinois, paradoxe à la vérité peu original, est que, s'il est né d'une assomption à la fois subie et voulue du monde extérieur, il n'a pas eu de tâche plus urgente, après son arrivée au pouvoir en 1949, que de s'entourer d'une clôture tendanciellement totale. Cet empressement n'était pas seulement l'effet mécanique de la victoire d'authentiques nationalistes. Car le PCC ne se contentait pas d'abolir les concessions de toute sorte que l'Etat " nationaliste " Guomindang avait abandonnées aux " puissances ». L'ambition, désormais, était d'interdire toute relation non contrôlée avec l'extérieur. La Chine passait de la définition traditionnellement culturelle de la frontière à une définition non pas administrative, mais idéologique et politique.

Cette transition brutale offre un point de vue sur le nouveau régime communiste. Elle confirme que ses principes et ses objectifs communistes sont totalitaires en nature. Car la fermeture communiste est en principe une limite dynamique qui inscrit un régime dans un camp, celui de la révolution, pour l'opposer à l'autre, celui du capitalisme, et pour lui permettre de progresser dans son sein. Mais la nature totalitaire du régime rend cette fermeture statique. Celle-ci vise, dès lors, moins la conquête extérieure que la domination intérieure. Ce n'est pas une frontière de colonisation mais une protection. Non que la nature totalitaire d'un régime l'engage au pacifisme. Au contraire, comme le notait Denis Delbourg à propos d'Orwell, le totalitarisme a besoin d'une guerre lointaine mais peu risquée et peu coûteuse, d'« une rage perpétuellement renouvelée mais perpétuellement contenue ", qui légitime l'incessant renouvellement des efforts de totalisation du corps social : tel est le sens du slogan de 1984 : «La guerre, c'est la paix »'.

1. Delbourg (Denis), "Guerre et stratégie », Le Genre humain, 9, 1983, p. 209-229. 
En fait, dans le même temps où le nouveau régime ferme ses frontières, il inscrit la clôture au cœur de l'espace intérieur en le cloisonnant. Ce faisant, il abolit des ouvertures qui avaient intégré l'espace chinois dans l'espace cardinal et permis l'importation de modèles révolutionnaires : l'ouverture de la frontière - la majorité des dirigeants nationalistes chinois (sauf Mao, nous y reviendrons) ont fait leurs classes à l'étranger - et l'ouverture de l'espace urbain sur les campagnes - le sauvetage puis la victoire de la révolution chinoise sont le résultat d'un aller-retour des villes aux campagnes. La clôture est également sociale. Le régime découpe deux univers distincts : le «peuple » et ses «ennemis ", qui sont annihilés ou enfermés. Le " peuple " lui-même est réparti en couches sociales disposées en cercles concentriques autour du Parti, entre lesquelles la circulation est découragée. Entre les individus euxmêmes, la communication est idéologiquement dé-ligitimée et pratiquement réduite par la mobilisation verticale des énergies et des sentiments que le parti réalise.

La clôture totalitaire tend même à extraire la Chine du temps mondial. La révolution chinoise était sortie de l'idée que le pays devait être « remis à l'heure du monde». Mais le tocsin de Moscou est luimême devenu cadran. Désormais, tous les grands événements prennent sens sur la grande horloge du Kremlin. Celle-ci n'ignore pas le temps des adversaires, elle en tient suffisamment compte pour protéger les intérêts fondamentaux de l'URSS - on sait quelle prudence concrète Staline a constamment montrée face aux menaces extérieures. Mais, en interprétant le temps mondial, elle le décale et inspire des horaires locaux qui peuvent s'en éloigner plus encore, et même des " sorties du temps " locales. Ainsi, après la fin de la guerre de Corée (1953), la Chine s'engage dans la durée de la «construction de socialisme». Désormais, le monde extérieur global et réel cesse de constituer une détermination décisive pour elle. Les dirigeants de Pékin règlent le rythme de leur action sur deux déterminations abstraites : le "rapport de forces international » et la «situation de la lutte des classes». En fait, l'« heure de Pékin " est partiellement sortie du temps mondial. Elle scande une "nouvelle époque » qui est celle de la «Nouvelle Chine» où se forge un « homme nouveau ».

Clôture de l'espace, clôture du temps : ces ambitions inouies suggèrent immédiatement les arguments de réalité classiquement opposés à la problématique du totalitarisme. De fait, par définition, toute histoire totalitaire est l'histoire d'un échec. Mais de l'échec du projet totalitaire. Non seulement ce projet comprend une prétention typique à recouvrir le réel social et naturel, mais il a reçu un degré parfois considérable d'effectivité, notamment au moment des grandes terreurs. Ainsi, dans un premier temps, le PCC est parvenu à modifier et à quadriller partiellement l'espace intérieur chinois. Ecoutons ce que déclare en 1964, en plein cœur de la domination maoïste, le paysan d'un village situé dans la partie traditionnellement la plus « ouverte » sur l'étranger de la province de Guangdong, à mi-chemin de Hong Kong et de Canton : "Ainsi, vous êtes de Canton ? Où est Canton? Est-ce en Chine ou en Amérique ? 
Est-ce aussi grand que notre village ? "'. Ce qui impressionne, dans cette question, ce n'est pas l'ignorance - après tout, ce paysan connaît l'existence de l'Amérique, sans doute grâce à la propagande officielle ; c'est qu'il confond dans un même "étranger ", et Canton, et l'Amérique, témoignant ainsi de la puissance de la clôture qui isole désormais les collectivités villageoises.

L'échec des entreprises totalitaires est largement commandé par l'orientation et les contradictions des modèles qui les animent successivement. S'il existe une histoire totalitaire, et une histoire de la clôture totalitaire en Chine, c'est en large partie à cause du rapport ambigu et fluctuant qui a uni les communistes chinois au modèle soviétique qu'ils instrumentalisaient.

\section{LE MODÈLE SOVIÉTIQUE, INSTRUMENT ET LIMITE DE LA CLÔTURE}

Car l'alliance des années 1949-1959 est bien loin d'épuiser la réalité des rapports sino-soviétiques. Ces rapports sont, beaucoup plus globalement, ceux du maître et du disciple - un maître dont l'enseignement change, un disciple dont l'indiscipline s'affirme. La clôture totalitaire va être menacée par les contradictions et par l'évolution de cette relation.

Dès l'abord, le projet d'instrumentalisation, et sa difficulté, sont résumés à Pékin par un concept qui va servir de titre à la revue du comité central du PCC: Xuexi, qui signifie à la fois «apprendre » et " s'inspirer de ». Mais, dès le début aussi, il apparaît que les conditions de l'instrumentalisation sont devenues plus difficiles. Dès lors qu'il a physiquement conquis le territoire chinois, le $\mathrm{PCC}$ se trouve concrètement au contact direct du " grand frère », de ses ambitions territoriales et de sa soif de domination. De plus, le prix de l'aide soviétique est accru par l'intense bipolarisation internationale et par la nature de l'œuvre intérieure à accomplir, une «construction économique » dans laquelle le PCC ne possède aucune expérience.

Conscients de ce déséquilibre et des dangers qu'il implique, les dirigeants chinois s'efforcent de maximiser les avantages de leur relation avec Moscou tout en minimisant son influence sur leur pouvoir. Mais, de son côté, l'allié métropolite fait payer au prix fort son assistance et ses leçons. Le marchandage est permanent. Ainsi, les communistes chinois vont chercher à Moscou l'aide économique qui les remboursera de leur intégration au "camp socialiste". Mais Staline sait trop bien qu'ils ne possèdent pas de véritable alternative : ils reviennent avec des promesses inférieures à leurs attentes et surtout doivent bientôt payer le prix du sang par une intervention dans la guerre de Corée. Aussi bien ne se gênent-ils pas pour mettre en évidence l'originalité de leur expérience révolutionnaire $^{2}$. A la mort de Staline, la faiblesse interne des nouveaux

1. Chan (Anita), Madsen (Richard), Unger (Jonathan), Chen village, Berkeley, University of California Press, 1984, p. 15.

2. Hu Chi-hsi, Pékin et le mouvement communiste indien, Paris, Presses de la Fondation nationale des sciences politiques, 1972. 


\section{Jean-Luc Domenach}

dirigeants soviétiques modifie pour un temps le rapport des forces. L'aide soviétique augmente, et la Chine peut jouer à son avantage le rôle de «brillant second» de la diplomatie soviétique. Mais à peine le pouvoir de Khouchtchev est-il consolidé, en 1957, que Moscou présente de nouvelles exigences stratégiques inacceptables pour un régime communiste indépendant.

Des deux côtés, donc, il y a double jeu sur tous les tableaux à la fois. Ce double jeu est mutuellement utile parce que chaque partenaire offre à l'autre quelque chose d'essentiel et qui lui manque complètement. A Moscou, Pékin offre un brillant élève du Tiers Monde. A Pékin, Moscou offre une voie d'accès à la modernité, celle de l'industrialisation planifiée, et cela, dans des conditions d'indépendance, qui donc n'obèrent pas l'avenir.

La clôture totalitaire vit et souffre de ce double jeu. D'un côté, et c'est l'essentiel, elle est légitimée par la notion de "camp socialiste " et aussi, d'ailleurs, organisée grâce aux conseils soviétiques. D'un autre côté, le modèle soviétique lui-même prévoit un certain nombre de médiations juridiques, administratives et politiques qui sont autant de limites à la définition d'une clôture totale. De plus, la nécessité d'une politique étrangère partiellement découplée de Moscou - c'est-à-dire d'une politique régionale tirant profit des positions et de l'influence chinoises en Asie - implique le maintien d'un minimum de relations économiques et sociétales avec les pays voisins. Elle implique surtout des relations suivies avec l'archipel des communautés chinoises d'outre-mer. De là un flux permanent, jamais totalement assimilable, d'hommes et d'idées qui relient la Chine communiste aux capitalismes renaissants d'Asie du SudEst. Pékin n'a jamais aussi complètement que Moscou rompu les liens avec son émigration capitaliste : la stratégie structurellement prudente poursuivie depuis le début dans l'affaire de Hong Kong est de ce point de vue significative.

Mais ce sont les relations avec le bloc soviétique qui portent la contradiction au cœur du système. Car les métastases changent rapidement de nature. Staline et ses féaux est-européens étaient de difficiles partenaires. Du moins représentaient-ils pour la Chine une option - et un danger - clairs et uniques. Staline mort, cependant, le modèle soviétique paraît évoluer politiquement et diplomatiquement vers l'Ouest. Les troubles est-européens montrent les dangers de cette évolution. Elle vient jeter le doute dans un régime chinois où le modèle stalinien s'implante tout juste. La déstalinisation khrouchtchévienne séduit certains collègues de Mao Tse-tung et inspire l'apparition d'une étroite frange réformatrice dans le parti. La littérature du dégel et les dissidences est-européennes constituent pour les intellectuels chinois un exemple qui sera suivi dès 1957. Mais Mao, pour sa part, conclut de ces influences que le modèle soviétique est en voie de perdre ses vertus propres. Car il est bien vrai qu'après avoir été source de légitimation et de consolidation le modèle soviétique, en se déplaçant, contribue à fragmenter le pouvoir de Pékin. La clôture totalitaire s'est avérée la plus fragile par le lien qui la fondait. Elle a laissé passer des influences qui ont précipité une crise d'origine 
interne dont est sortie, en 1958 , la première grande aventure maoïste, le Grand bond en avant.

\section{L'ÉCHEC DE LA RECOMPOSITION MAOÏSTE}

L'entreprise maoiste est d'abord un effort de recomposition de cet Est révolutionnaire que le " révisionnisme » de Khrouchtchev décomposait. Il s'agit de transférer à Pékin le pôle de la révolution mondiale. Par le Grand bond en avant, la Chine se saisit de l'exemplarité révolutionnaire : chez elle, le communisme est moins éloigné encore qu'en URSS. Puis, la grande polémique sino-soviétique démontre la fidélité chinoise au dogme et la trahison de Moscou. Avec la Révolution culturelle, enfin, apparaît la version chinoise de l'Evangile communiste : le "précieux livre rouge ». Nul besoin d'un changement de contenu, puisqu'il faut au contraire démontrer une plus grande fidélité : le "petit livre rouge » réincorpore les principaux thèmes de la vulgate stalienne, en leur ajoutant simplement des formules prétentieuses sur la grandeur de la Révolution chinoise. Ce qui est vraiment nouveau, c'est ce que des publicitaires appelleraient le «produit », c'est-à-dire le format - celui d'un guide pratique -, la couleur rouge et la signature : en publiant ses Citations, Mao devient le Staline des temps modernes. La Révolution culturelle, déclenchée pour mettre fin aux entraves intérieures que Mao supportait encore, est aussi une épreuve de purification pour le communisme chinois et un exemple pour le monde. La « lutte révolutionnaire » est pour les Gardes rouges l'occasion de s'épurer et d'épurer le parti. La Chine tout entière doit mimer le retour à la pauvreté, à la pureté des bases rouges : pour se saisir du flambeau de la révolution mondiale, le PCC doit démontrer qu'il en est le plus digne.

Dès lors, la propagande chinoise peut rayonner à l'extérieur. Dans les années cinquante, elle rediffusait dans le Tiers Monde - principalement en Asie - des programmes qui étaient pour l'essentiel ceux de Moscou, exception faite de certaines inflexions piquantes. Au début des années soixante, Pékin prend le monde à témoin de la trahison soviétique. A partir de 1965-1966, commence la prédication. La Chine maöste se donne en exemple. Cet exemple est à la fois celui de la révolution sans compromis, d'une révolution continuée après la prise du pouvoir et qui vise la transformation idéologique : tous éléments extraits de la vulgate stalinienne, mais qu'elle s'approprie. Ce rayonnement se veut universel. Il irradie les campus occidentaux et tente de percer les brouillards esteuropéens. Dans le courant de 1968, les «larges masses " pékinoises défilent pour soutenir les étudiants français puis pour protester contre le coup de force "social-impérialiste » en Tchécoslovaquie. Mais, typiquement, c'est surtout au Tiers Monde que l'on s'adresse, parce que l'on estime que les «campagnes du monde " finiront par « encercler » ses métropoles : le modèle intérieur de la prise du pouvoir est étendu au domaine international'. 1965.

1. Lin Biao, "Vive la victorieuse guerre du peuple", Quotidien du peuple, 3 sept. 
Cette volonté de rayonner sur le monde n'entraîne pas d'assouplissement mais au contraire un durcissement de la clôture totalitaire. Le rayonnement maoïste, c'est le rayonnement du diamant. La Chine est figée dans son exemplarité, enfermée dans sa perfection. Mao oppose un réseau étanche aux infiltrations en provenance de l'Ouest. La frontière de Hong Kong est désormais pratiquement hermétique : seuls la franchissent les diplomates et les convertis. Les liens avec les communautés chinoises d'outre-mer sont le plus souvent « révolutionnarisés » ou rompus. Les centaines de milliers de patriotes naïfs rentrés au pays dans les années cinquante sont désormais persécutés.

Des purges frappent ceux qui avaient été en relation étroite avec Moscou. Les derniers témoins de l'influence étrangère sont recouverts par les slogans nouveaux. La rue qui conduit à l'ambassade d'URSS est rebaptisée "rue de l'antirévisionnisme». Et, symbole plus significatif encore, la Shanghaï des concessions étrangères est retournée en un bastion de la Révolution culturelle. Bref, la clôture n'est plus seulement protection. Elle concourt au rayonnement extérieur en délimitant le territoire de l'aventure sacrée. Car la révolution chinoise ne conquerra pas le monde par la guerre, mais par l'exemple. Quelques mois avant de déclencher la Révolution culturelle, Mao décide de ne pas augmenter son engagement militaire dans un Vietnam Nord qui, désormais, ploie sous les bombes.

Tout aussi significatif que cet effort, cependant, est l'échec qu'il subit, dont les signes apparaissent rapidement : dès 1965, le massacre des communistes indonésiens constitue une grave défaite politique pour la Chine maoïste. Echecs intérieurs et extérieurs s'additionnent. Si la recomposition maoïste échoue, c'est d'abord parce qu'elle est idéologiquement incohérente.

En premier lieu, Mao n'a jamais concrètement remis en cause les privilèges budgétaires de l'industrie lourde". "Marcher sur les deux jambes » n'était au mieux qu'une procédure destinée à investir plus massivement encore les ressources du monde rural dans l'industrialisation. Au pire, ce n'était qu'un slogan. La propagande ruraliste adressée au monde extérieur a fini par s'effacer devant cette réalité.

C'est essentiellement dans le domaine superstructurel que Mao a innové. Car il a eu le courage de reconnaître une caractéristique banale du communisme au pouvoir, qui est la contradiction entre la nécessité politique de la mobilisation des masses et la bureaucratisation effective du parti. Mais les remèdes qu'il a successivement essayés ont échoué : placer autoritairement le parti à l'avant-garde de la mobilisation communiste (Grand bond en avant), le remplacer par des comités révolutionnaires (mais en conservant durant la Révolution culturelle l'exigence du monopole) ou rééduquer ses membres fautifs (mais il s'est alors heurté à la comédie du repentir). En fait, cet échec dérivait largement d'un doute universel, dans le parti et en dehors, sur la possibilité de ressourcer un

1. Aubert (Claude), "L'économie chinoise et le modèle soviétique ", Politique étrangère, 1, 1983, p. 51-62. 
régime dont les corruptions coïncidaient trop évidemment avec structures fondamentales.

L'échec intérieur permet de mieux comprendre les déconvenues extérieures. Tout d'abord, le réseau international de maoïsme n'est jamais parvenu à se constituer. Manquant de chaleur propre, les rayons de l'astre maoïste se sont le plus souvent confondus avec les attentes engendrées par des situations régionales. En Amérique latine, le maoïsme s'est greffé sur le guérillérisme castriste avant de s'y confondre presque complètement. En Afrique noire, le modèle chinois à oscillé entre l'aide aux guérillas, le remplacement de l'Union Soviétique, le soutien démagogique aux nationalismes antiblancs, une vague propagande sur l'« autocentrage » et un comportement diplomatique simplement opportuniste ${ }^{1}$. En Europe, le maoïsme a continuellement hésité entre l'archéo-stalinisme et une sorte d'anarchisme. En Asie, enfin, l'influence maö̈ste a simplement épousé les contours de l'influence chinoise, reculant chaque fois qu'elle rencontrait des régimes solides (au Japon, par exemple) ou une riposte soviétique décidée (au Vietnam).

Le rayonnement du maoïsme a aussi beaucoup pâti de la faiblesse de la puissance chinoise. Sauf dans les zones temporairement négligées par l'URSS - en Afrique, par exemple, jusqu'au début des années soixante-dix -, la Chine n'est jamais parvenue à se saisir du rôle de protecteur de la révolution : elle en avait de moins en moins les moyens économiques et militaires. Capable de défaire l'armée indienne en 1962, la Chine est dissuadée par la menace soviétique d'intervenir dans le conflit indo-pakistanais de 1965. L'escalade américaine au Vietnam fait apparaître de plus en plus nettement la supériorité soviétique : après avoir vaincu la France grâce à l'aide chinoise, Hanoi vaincra les EtatsUnis grâce à l'aide soviétique. Pendant la deuxième partie des années soixante et la première partie des années soixante-dix, l'écart de puissance ne cesse de se creuser entre le Chine de Mao et les deux Super-Grands. Cet écart de puissance devient manifeste à partir des incidents sur l'Oussouri, en 1969 : désormais, la menace soviétique est telle que, tout en poursuivant sa prédication révolutionnaire, la Chine se trouve contrainte de la délégitimer en recherchant un surcroît de protection du côté de l'Ouest.

Mais il n'est pas suffisant de dire que Mao n'a jamais eu les moyens de sa politique. En tout état de cause, il n'a pas augmenté l'engagement matériel de la Chine à l'extérieur en proportion de son engagement idéologique. Il a plutôt réduit le soutien apporté au Vietnam, et il ne semble pas avoir apporté d'aide massive à aucune guérilla. Là se trouve la limite des études qui croient découvrir, dans la Chine maoïste, une correspondance étroite entre le degré de mobilisation à l'intérieur et le degré $\mathrm{d}^{\prime}$ " hostilité » de la politique étrangère chinoise ${ }^{2}$. Le bellicisme

1. Laïdi (Zaki), Les contraintes d'une rivalité, les super-puissances et l'Afrique (19601985), Paris, La Découverte, 1986, p. 49-55.

2. Kuang-Sheng Liao, «Linkage politics in China : internal mobilization and articulated external hostility in the Cultural Revolution, 1966-1969 ", World Politics, juillet 1976, p. 590610. 


\section{Jean-Luc Domenach}

du modèle maoïste était essentiellement verbal. La Chine populaire n'a fait la guerre qu'avant ou après la haute époque maoïste. On peut estimer que Mao faisait de nécessité vertu quand il assurait que l'essentiel était d'indiquer la «voie» et, pour les «peuples en lutte», de «compter sur leurs propres forces ». Et il est de fait que le rayonnement idéologique de Pékin a été très supérieur à ce que les moyens concrets engagés permettaient d'escompter.

Cependant, la façon dont ce principe a été concrètement appliqué fait naître un soupçon : que, dans son effort de recomposition révolutionnaire, Mao Tse-tung ait réinvesti des éléments traditionnels de la culture politique chinoise. Car, dans la pratique maoïste des relations extérieures, réapparaît un cinquième point cardinal auquel Georges Boudarel fait référence à propos du Vietnam : celui de la centralité de la Chine' '. Qu'à la différence de Staline Mao n'ait jamais été intéressé par la création d'un grand appareil international, cela éclaire le fait que la « voie » proposée aux «peuples du monde » a toujours été mécaniquement identifiée avec l'expérience ou avec les intérêts stratégiques de la Chine. L'altruisme introverti de Mao évoque irrésistiblement les traditions impériales chinoises.

Tout se passe en fait comme si, pour Mao, la clôture était un objectif bien plus important que le rayonnement qu'elle devait faciliter. La Chine des années soixante, fièrement arc-boutée sur sa misère, a remboursé rubis sur l'ongle ses dettes à l'URSS, fabriqué la bombe A puis la bombe H. En 1976 encore, après l'épouvantable tremblement de terre de Tangshan, Pékin a refusé avec hauteur les propositions d'aide étrangères.

Mais ces succès évoquent surtout les arrangements symboliques de la tradition. Dans les faits, au moment même où elle atteignait son acmé, la clôture totalitaire a été frappée de plein fouet par deux échecs. Le premier, induit par la supériorité militaire de l'URSS, est, en 1968-1972, l'ouverture stratégique sur l'Occident. Menacé dans son existence même, le régime se tourne vers l'un de ses ennemis. Dès lors, Zhou Enlai perce sous Mao Tse-tung et renaît une logique d'Etat qui s'était effacée. Pour nouer des relations avec les Etats-Unis, il faut rétablir une diplomatie : donc, restaurer partiellement la dualité entre l'Etat et le parti, et réintroduire à l'intérieur du pouvoir des médiations qui le bornent. Il faut également mimer au moins des échanges intersociétaux - commerciaux, culturels, humains. Dès lors, le monde extérieur retrouve un statut cognitif : la géographie est, après l'archéologie, l'une des premières «sciences » réhabilitées après le grand vide des années 19661969. Il retrouve également une réalité humaine, à l'intérieur, par le biais des Chinois d'outre-mer, qu'il n'est plus possible de repousser, car ils sont le symbole du lien avec l'Occident. La visite en Chine du

1. Boudarel (Georges), «The Vietnamese diaspora - postwar Vietnam = ideology and action », Institute of Development Studies, The University of Sussex, septembre 1985, art. non publié. 
président Nixon en février 1972 a donc marqué une date considérable : on comprend la stupéfaction du bon peuple pékinois.

La clôture totalitaire a également été victime d'un deuxième échec, le plus intérieur qui soit. Son efficacité dépendait largement de l'intensité de la mobilisation sociale. Or tout indique que la population chinoise a beaucoup plus mal supporté les désordres politiques de la Révolution culturelle que la catastrophe économique du Grand bond en avant. Que le pouvoir réprime ou se trompe, c'était après tout dans l'ordre traditionnel des choses. Mais qu'il se fragmente et cesse d'assurer la sécurité des citoyens, voilà qui n'était pas admissible : le désordre a bien plus scandalisé que l'utopie totalitaire. Puisqu'il faut obéir, les Chinois défilent et annoncent les slogans officiels. Mais désormais, ils pensent à autre chose. La Chine reste couverte de drapeaux rouges, mais le rouge ne tient plus. Il se déssèche, il se craquèle avant de partir par plaques entières. Les Chinois ont, au sens strict, l'esprit ailleurs. Ils pensent à leurs activités privées, à leur famille. Et, partout où la notion a un sens, ils pensent à l'étranger. Car l'étranger est la figure concrète du rêve. L'étranger ? A la vérité, tous les étrangers. Il y a eu des Gardes rouges pour fuir en URSS et, plus réellement surprenant, en Corée du Nord. Car, au moins pour les plus désespérés, tout, désormais, vaut mieux que la comédie quotidienne qui se joue en Chine. Mais, pour la grande masse des citadins, l'étranger prend surtout le visage de l'Occident capitaliste. Tout y concourt. Les souvenirs, d'abord, embellis par la misère des temps. Les allées et venues des Chinois d'outre-mer, ensuite. La proximité de Hong-Kong, enfin, Hong-Kong qui ressemble à un grand vaisseau de luxe amarré face aux côtes chinoises, Hong-Kong qui draine des contingents de fuyards de plus en plus massifs dans les années 1971$1976^{\prime}$. Au moment où le peuple chinois découvre sa misère, il apprend la prospérité des capitalismes extrêmes-orientaux : Taiwan, Corée du Sud, Japon bientôt... Et, suivant la bonne habitude qui lui avait été enseignée par la propagande officielle, il compare. C'est bientôt toute la Chine « utile » qui, en esprit, s'évade vers les féeries de l'Occident capitaliste. Le quotidien le plus attentivement lu est une feuille de traductions de la presse étrangère ${ }^{2}$. Au début de 1979, pressé par le président Carter de faciliter la circulation des personnes, Deng Xiaoping mettra immédiatement un terme à la bonne volonté de son partenaire en lui demandant combien de millions de Chinois les Etats-Unis sont prêts à accueillir... Avant même d'être abaissée par le pouvoir lui-même, la clôture totalitaire avait largement disparu de l'esprit des Chinois.

1. Domenach (Jean-Luc), «Y a-t-il une frontière chinoise ? La circulation des informations entre Hong-Kong et la Chine ", Revue française de science politique, 29(4), août-octobre 1979, p. 847-876.

2. Kinsella (Ivan), "Echos de la presse étrangère en Chine : le Cankao Xiaoxi ", Cahier d'études chinoises, 3, mai 1985, p. 9-62. 
Mais cette évolution ne deviendra manifeste qu'après un changement politique de grande ampleur, qui s'est opéré entre 1976 et 1982 , et que l'on peut résumer par la victoire de la ligne politique de Deng Xiaoping. Cette ligne ajoute à la priorité traditionnelle de tout régime communiste - conserver le monopole du pouvoir politique - un deuxième impératif urgent : moderniser le pays afin de le hausser au niveau des plus grandes puissances de ce monde. Son application se déploie donc dans deux champs distincts, celui du pouvoir et celui de l'économie. Dans le premier, on assiste à l'extinction des ambitions innovatrices du maö̈sme et à un retour aux institutions et aux méthodes de direction éprouvées dans les années cinquante, c'est-à-dire pour l'essentiel au modèle soviétique tempéré par la disparition de la terreur. En revanche, le domaine économique est abandonné à une gestion bien plus pragmatique que réformiste, qui mélange toutes les solutions possibles, y compris celles venues du monde capitaliste, dans des mixtures qui varient suivant les équilibres politiques et la connotation idéologique des problèmes. Si les monopoles centraux du pouvoir demeurent entiers - ce qui lui laisse une capacité de réversion en principe totale -, ses monopoles subsidiaires (organisation économique, communication) sont fortement érodés.

Cette évolution possède une traduction «cardinale». Tout d'abord, Pékin tire les conclusions de son échec à « recomposer » l'Est en rentrant, sans commentaires, dans la matrice institutionnelle soviétique. Après l'éviction des partisans de Mao Tse-tung, le groupe dirigeant s'est naturellement trouvé formé d'hommes qui ont dirigé ou appris à diriger suivant les critères soviétiques et, bien souvent, face aux Soviétiques, dans les années cinquante. Le grand maître de l'économie est Chen Yun, l'homme du premier plan quinquennal. Hu Yaobang, l'actuel secrétaire général du PCC, a fait ses classes à la tête de la Ligue des jeunesses et dans le réseau international des mouvements de jeunesse communistes ${ }^{1}$. Li Peng, aujourd'hui membre du bureau politique, aurait été quelques mois condisciple de Gorbatchev à Moscou. Et l'actuel ministre de la Sécurité publique, Ruan Chongwu, est sorti en 1957 ... de l'Institut de mécanique automobile de Moscou $^{2}$.

En même temps, le champ de la modernisation est désormais largement ouvert à l'Occident. Les pays de l'Ouest ne sont plus seulement des partenaires diplomatiques, ce sont aussi des partenaires économiques et, dans une mesure limitée, sociaux. Le rêve de l'Occident n'est plus seulement populaire, il est officiel. C'est au Japon que Deng Xiaoping déclare en 1978: "J'ai enfin compris ce que signifie le terme de modernisation ", quelques semaines avant de coiffer le chapeau texan

1. A l'occasion de la visite en Chine d'un important responsable est-allemand, en décembre 1985, Beijing Information signalait benoîtement que "Honecker ... a eu déjà des rapports étroits avec Hu Yaobang dans le mouvement international de la jeunesse " (Beijing Information, 30 décembre 1985, p. 8).

2. Beijing Information, 30 décembre 1985, p. 16. 
lors d'un voyage officiel aux Etats-Unis. Ce rêve, certes, nourrit des sentiments compliqués. Comme avant 1949, la supériorité de l'Occident inspire à la fois l'admiration et la haine. Les équipes sportives occidentales savent qu'il est difficile de gagner un match en Chine. La plus importante difficulté politique rencontrée par Deng Xiaoping depuis 1982 a été une « campagne contre la criminalité », habilement détournée par ses collègues «contre la pollution spirituelle de l'Occident». Mais peu importe. La supériorité de la technologie occidentale est unanimement reconnue. Pour réaliser sa modernisation, la Chine l'importe et l'assimile autant que possible. Ses étudiants dans les universités occidentales seraient aujourd'hui plus de quinze mille, soit plus de deux fois le nombre de ceux qui fréquentaient les instituts est-européens dans les années cinquante. D'après un bruit persistant, plus de la moitié des membres du comité central du PCC compteraient parmi leurs parents un boursier à l'étranger.

Il existe donc une intéressante asymétrie entre les relations que la Chine entretient aujourd'hui avec l'Est et avec l'Ouest. Le cour du régime politique est rentré dans l'Est soviétique. En revanche, ses périphéries s'ouvrent à l'Occident. Périphérie géographique : le Sud de la Chine est infiniment plus « ouvert» que le Nord et l'Ouest. Périphérie sociale : les couches corrodées par l'Óccident sont étroites et fragiles (exportateurs, intellectuels) ; les cadres ne sont que partiellement touchés, et leurs enfants ont tout le temps de revenir aux sains principes pour monter dans l'appareil. Périphérie politique, enfin : ce sont les secteurs les moins influents qui traitent le plus souvent avec l'Ouest - l'Etat plus que le parti, les départements techniques plus que les départements politiques. La diplomatie du PCC, elle, réintègre insensiblement le monde communiste. En fait, le changement le plus significatif se situe en amont et en aval de cette divergence : dans le changement du rapport entre la Chine et le monde. Lorsqu'il définit les changements récents comme la " deuxième révolution chinoise ", Deng Xiaoping est peut-être exact. Car, comme les nationalistes chinois du début du siècle - dont il était, d'ailleurs -, il s'efforce d'abolir la clôture qui sépare la Chine du monde afin de ramener son pays dans la concurrence internationale. Cette décision, certes, est le produit d'un processus interne dans lequel la décomposition du pouvoir maoïste a joué un rôle décisif que l'étranger n'a guère compris, et peu influencé. Mais cette décomposition et le choix de Deng ont été hâtés par la comparaison permanente entre les échecs chinois et les succès étrangers, qui constitue l'arrière-plan des ultimes disputes de succession en 1975 et $1976^{1}$. La ligne pragmatique l'a emporté pour des raisons purement internes. Mais elle n'aurait jamais vu le jour si n'était pas devenu apparent un dramatique différentiel de puissance et de niveau de vie entre la Chine et ses principaux partenaires.

La reconnaissance que le monde extérieur est aussi le monde de la Chine est donc une inspiration permanente de la ligne pragmatique. La

1. L'un des épisodes les plus vifs de la lutte de succession a été provoqué par le discours de Zhou Enlai à l'Assemblée nationale, qui, en janvier 1975, préconisait de ramener «l'économie chinoise aux premiers rangs du monde". 
part du commerce extérieur dans le PNB chinois est passée de $5 \%$ à $10 \%$ entre 1978 et $1984^{\prime}$. Les universitaires chinois opèrent une entrée progressive dans des domaines comme la sociologie, le droit et la science politique, où les dogmes marxistes se trouvent inévitablement mis en question $^{2}$. Mais le signe le plus important de ce retour dans le monde est l'évolution à la fois pratique et sémantique de la diplomatie chinoise. Jusqu'au début des années quatre-vingts, celle-ci avait toujours été une diplomatie du camp, défendant successivement le socialisme, les campagnes du monde puis les puissances subordonnées contre la malignité des forces dominantes : impérialisme, super-puissances, social-impérialisme. Aujourd'hui, la pratique diplomatique chinoise est beaucoup plus utilitariste : elle vise à faciliter la modernisation du pays en multipliant ses partenaires économiques et en assurant sa sécurité régionale. Son action n'est plus défensive, mais positive : pour elle, le monde n'est plus seulement dangers, mais ressources. Et son discours, lui, a plus encore évolué. Au moment où la crise économique internationale déchaîne les égoïsmes, le discours chinois prend en charge les intérêts de l'univers : il prône avec une apparente naïveté la paix, l'entente, la coopération régionale et internationale... Nul doute, certes, que ce langage possède une fonction politique précise : faire grandir la popularité de la Chine et lui donner une sorte de rôle tribunicien dans les arènes internationales. Nul doute, aussi, qu'il reflète le changement de statut du monde extérieur dans la politique chinoise et l'idée que ce dernier est utile à l'évolution de la Chine.

La preuve qu'il s'agit d'une idée fortement ancrée est que les mêmes dirigeants qui avaient édifié la clôture des années cinquante ont accepté le retour du monde en Chine et, dans une certaine mesure, la cardinalisation de l'espace intérieur chinois. Ils sont conscients des dangers induits par l'écart de puissance entre la Chine et ses grands partenaires. La séduction des pays occidentaux est explicitement perçue comme une «pollution». Mais c'est un mal inévitable qu'ils estiment possible de marginaliser. Les dirigeants chinois ont eu tout le temps de réfléchir à l'erreur d'analyse qu'ils avaient commise à propos de l'URSS dans les années soixante : celle-ci leur a donné la preuve qu'il est possible de combiner sans dommage ouverture technologique et fermeture politique. Ils sont certes contraints par leur pauvreté et par l'ampleur de l'échec maoiste d'ouvrir plus encore leur espace intérieur. Mais, pour limiter les effets de cette ouverture, ils travaillent habilement à diviser et opposer entre elles les influences occidentales. A la tactique classique qui consiste à opposer les Etats-Unis à l'Europe et les pays européens entre eux, ils en ajoutent une autre, qui est le recours financier et technologique aux Chinois d'outre-mer, auxquels sont affectés, suivant les cas, les rôles délicats et décisifs d'éclaireurs, d'intermédiaires ou de leurres. Mais la solution centrale est plus simple encore. Elle consiste à replier autour

1. Sur ce point, voir Godement (François), « La Chine et l'Asie », Politique étrangère, 4, 1985, p. 849-870.

2. De ce point de vue, la lecture de la revue Recherches de sciences politiques fondée en 1985 est instructive. 
du cercle du pouvoir la clôture qui entourait et quadrillait autrefois l'espace' social. Le pari consiste à maintenir par là une capacité de réversion en principe complète et à espérer que son maintien suffira à éviter de s'en servir '.

Les changements subis par l'espace intérieur chinois montrent cependant que la prise de risque est considérable. La seule partie qui soit totalement à l'abri des regards étrangers est le lieu du pouvoir : à l'image de ce comité central logé à l'abri des murs millénaires de la Cité interdite (mais à quelques centaines de mètres des flots de touristes). A l'inverse, Canton et une partie croissante de la Chine du Sud sont de plus en plus polarisés par Hong-Kong. Mais le plus significatif est l'apparition d'un entre-deux géographique et social où les influences s'entre-mêlent et se corrodent. Dans beaucoup de campagnes, l'affaiblissement du pouvoir laisse en présence le retour des coutumes ancestrales et l'arrivée d'une modernité à connotation étrangère. En même temps que l'on reconstruit les temples ancestraux, la télévision se répand bien avant la cuisinière à gaz. Or la télévision, ce n'est pas seulement un regard sur l'étranger, c'est bien souvent le regard de l'étranger : d'après une enquête réalisée en 1985 parmi sept stations de télévision en Chine méridionale, 21 des 22 émissions diffusées en fin de semaine étaient produites à HongKong ou au Japon ${ }^{2}$. Le plus souvent, l'étranger est capitaliste, mais il existe des exceptions : au Nord-Est, où les points de passage vers l'URSS ont été réouverts, et dans l'Ouest désertique où se combinent l'attrait économique des Républiques soviétiques d'Asie centrale et l'attrait religieux des grands courants islamiques. A la ville, on assiste à une sorte de « corps à corps » entre deux partenaires dont chacun se coule dans les faiblesses de l'autre. Bénéficiant de son monopole et de sa vigueur économique renouvelée, le pouvoir communiste abat les quartiers anciens, construit de grands ensembles urbains et, par une surveillance vigilante, cantonne les dissidences politiques dans la nuit du champ social ${ }^{3}$. Mais les influences de l'étranger se répandent dans les creux de l'action du pouvoir : le vêtement à l'occidentale remplace progressivement l'uniforme totalitaire, les loisirs s'individualisent et la réforme vient battre les murailles des grands "donjons » industriels du régime ${ }^{4}$. La tradition, au contraire, s'arc-boute sur son bastion familial. Bref, l'espace social se « cardinalise ».

1. Domenach (Jean-Luc), «La Chine ou les aléas du totalitarisme ", in Madeleine Grawitz et Jean Leca (sous la direction de), Traité de science politique, Paris, PUF, 1985, tome 2, p. 238-267.

2. Beijing Information, 2 décembre 1985, p. 7.

3. Domenach (Jean-Luc), "Politique souterraine et agitation sociale dans la Chine post-maoïste ", in Claude Aubert, Yves Chevrier, Jean-Luc Domenach, Hua Chang-Ming, Roland Lew et Wojtek Zafanolli, La société chinoise après Mao, Paris, Fayard, 1986, p. 91129.

4. Chevrier (Yves), "Les réformes en Chine ou la stratégie du contournement », Politique étrangère, 1, 1985, p. 119-138. 
Au total, la trajectoire historique du communisme chinois peut d'abord être présentée sous l'angle des rapports entre la Chine et ce que Skocpol appelle le «temps mondial ». Née d'une volonté de resynchronisation de la Chine avec un monde dont la modernité se construisait sans elle, la révolution communiste cherche d'abord à s'en extraire pour accomplir son caractère totalitaire, mais elle se trouve finalement contrainte de se resynchroniser. Cette trajectoire suggère plusieurs séries de réflexions. La première porte sur la distinction entre "monde extérieur» et «temps mondial ». La première notion n'est pas sans signification pour un pays comme la Chine qui a longtemps pensé sa civilisation et son organisation politique comme spécifiques, et donc son rapport avec l'étranger en termes d'altérité ou d'identité. Mais elle n'autorise qu'une représentation indifférenciée du monde extérieur. Or l'élément décisif est qu'à compter du milieu du $19^{c}$ siècle le monde ne pouvait plus être représenté en Chine comme un espace extérieur et indifférencié car il possédait des pôles de puissance capables de s'imposer à l'Empire et de le pénétrer. La Chine devenait désormais partie d'un univers mondialisé, rythmé par ses plus grandes puissances. Elle n'était pas seulement entourée du monde extérieur, mais pénétrée par la dynamique qui l'animait, celle des plus forts, une dynamique de modernité orientée par l'idée du futur. Le temps qui s'impose à la Chine est donc désormais le temps implacable des différentiels de dynamisme. Dès lors que la Chine abandonne son temps cyclique pour reconnaître le temps mondial comme son propre temps, le rythme des plus forts lui apparaît comme une norme à atteindre. Toute la question, désormais, devient celle des durées transitoires, qui se répartissent inévitablement en deux catégories. La plus rationnelle est celle du «détour », de la «transition» par assimilation progressive de rythmes intermédiaires. Mais le chemin est inévitablement long, d'autant que les "puissances" elles aussi accélèrent: il faut beaucoup de patience au Mao d'avant 1949 pour admettre les « sinuosités » spatiales et temporelles de la révolution. Et la tentation reste perpétuellement présente, chez les dirigeants d'un grand pays que sa culture traditionnelle plaçait au centre du monde, du " raccourci » qui permettrait par un «bond en avant » rapide le « rattrapage» du retard chinois.

Ces deux durées charrient avec elles deux représentations du monde. La durée du Bond en avant que Mao a choisie en 1958 implique une conception de l'espace mondial comme recentré autour des capacités de la Chine : même le cinquième point cardinal revêt dès lors une importance décisive. En revanche, la durée de la transition, du détour, présente l'espace mondial comme à la fois gros de menaces et de ressources alternatives. C'est, tout d'abord, un champ de forces dont les dilatations et les contractions ouvrent et ferment des opportunités aux pays du Sud. Dans la durée du bond en avant, tout paraît possible : la Chine fraye son chemin à l'horizon de l'univers tout entier. En revanche, la durée du détour est celle des objectifs hiérarchisés, des offensives ponctuelles et des politiques régionales. Mais le monde est aussi un champ idéologique 
optionnel, il est le champ du choix entre les modèles en compétition, qui seront les modèles de la transition. De façon significative, ce champ est beaucoup plus limité que celui de la puissance. Alors que ce dernier laisse un certain rôle au pays de second ordre, le premier est un marché, une bourse qui ne retient que les valeurs les plus sûres, celles des plus puissants. Deux valeurs seulement ont été prises en compte par les nationalistes chinois, celles de l'Ouest capitaliste et de l'Est communiste. Ils n'ont jamais été sérieusement tentés par les valeurs des filiales européennes ou du Tiers Monde. Celles-ci, qu'elles fussent françaises, yougoslaves ou cubaines, n'ont jamais servi qu'à abriter temporairement des transferts d'investissements d'une valeur à une autre. En dernière analyse, donc, la bourse des valeurs est une traduction exagérée des rapports de forces concrets.

La cotation des valeurs dépend des performances des deux modèles en présence. En Chine, comme dans nombre d'autres pays du Tiers Monde, les relations internationales sont perçues comme une gigantesque compétition dans laquelle s'affrontent à la fois les forces et les méthodes des équipes en présence. Cette compétition n'existe évidemment qu'au travers des représentations qu'elle inspire, d'où l'importance décisive et par ailleurs bien connue du contrôle de l'information internationale. Le cas chinois, sans être nécessairement original, permet de préciser quelque peu la façon dont les résultats sont mesurés. Ainsi, première remarque, la compétition internationale est souvent représentée dans un code non politique. Par exemple, l'une des raisons de l'extraordinaire popularité du sport de haute compétition, et notamment des Jeux olympiques, est qu'il figure la compétition politique et idéologique dans un code aisément compréhensible du plus grand nombre. L'habileté des régimes communistes, y compris ceux du Tiers Monde, est de l'avoir compris.

Une deuxième remarque est que les comparaisons de niveaux de vie jouent également un rôle considérable, mais qu'en Chine au moins ces comparaisons sont surtout effectuées pour les pays comparables, c'est-àdire pour les pays voisins. Le citadin chinois n'a guère de moyens de mesurer la différence entre l'abondance soviétique et l'abondance occidentale - sauf à rêver. En revanche, la comparaison entre son propre revenu et celui des habitants de Taiwan, de Hong-Kong, de Singapour ou même du Japon est plus aisée, à cause de la proximité des civilisations et des modes de vie, et surtout à cause de la circulation des Chinois d'outre-mer. On peut se demander si ceux-ci n'ont pas été, depuis le milieu des années soixante-dix, les vecteurs d'une véritable opinion publique régionale en Asie orientale, une opinion publique qui, en marge des régimes politiques, a massivement opté pour le libéralisme économique.

En amont et en aval de l'opinion publique, en tout cas, les dirigeants chinois tiennent un compte précis des performances des pays voisins. Le Japon et, à un moindre titre, l'Inde sont pour eux des références permanentes - car c'est déjà par comparaison avec leurs collègues nippons et, à un moindre titre, indiens qu'ils avaient défini leurs choix. Il existe en tout cas un intéressant parallélisme entre les performances des 
partenaires régionaux de la Chine et les choix politiques successifs des dirigeants de Pékin. Quand en 1949 le communisme arrive au pouvoir en Chine et décide de "pencher d'un seul côté », la situation du Japon et de l'Inde inspire de graves inquiétudes - on l'a trop oublié aujourd'hui' '. Mais quand il se tourne vers l'Occident, à la fin des années soixante-dix, c'est en prenant acte de façon explicite des succès remportés par le Japon et les nouveaux pays industriels.

Cependant, c'est en dernière instance en Chine même que se forment les perceptions, que celles-ci soient le fait des populations ou des élites. C'est en Chine que s'opère la cotation des valeurs. Les performances des modèles en compétition ne prennent sens que par rapport aux besoins ressentis. Le choix effectué en 1949 est à ce point de vue significatif. Car, lorsqu'ils parviennent au pouvoir, les dirigeants communistes connaissent d'expérience la puissance matérielle et les ressources politiques de la démocratie américaine - la collaboration a été fructueuse entre Yanan et le commandement américain durant la guerre contre le Japon. Et pourtant, la volonté révolutionnaire qui les anime les contraint à pencher du côté du seul modèle révolutionnaire disponible, le modèle soviétique. Ils ne se tourneront partiellement vers l'Ouest qu'à partir de la fin des années soixante-dix, quand leurs échecs les auront conduits à ressentir de nouveaux besoins.

Une première leçon de l'expérience chinoise est donc que l'appel aux modèles exogènes, quoique très largement conditionné par l'environnement international et régional comme par le marché mondial des valeurs, dépend en dernière instance de perceptions et de choix intérieurs. S'il est vrai que le temps mondial ne peut être ignoré impunément, il est plus vrai encore que la durée historique de chaque pays détermine le dessin de la nécessaire re-synchronisation. En cela comme en d'autres matières se confirme le rôle des élites nationalistes du Tiers Monde. Le rôle historique des mouvements nationalistes n'aura pas seulement été la construction d'Etats-nations indépendants, mais également la détermination des choix de modernisation, et l'engagement des nouveaux pays indépendants dans des durées autonomes.

\section{L'ITINÉRAIRE INTÉRIEUR DU MODÈLE SOVIÉTIQUE}

Le trajet «chinois" du modèle soviétique confirme, complète et nuance à la fois cette conclusion. Il montre en effet, tout d'abord, que le modèle soviétique a subi de profondes modifications en Chine même. Dans un premier temps, il a été instrumentalisé, c'est-à-dire assimilé suivant des modes et à des fins internes. L'indépendance du processus d'instrumentalisation contraste alors avec le mimétisme volontariste des dirigeants de Pékin. Ou plutôt, c'est l'indépendance du processus qui autorise le mimétisme. L'ouvrier chinois des affiches de propagande a le

1. Verceil (Claude), "Les trois âges de l'Asie contemporaine », Défense nationale, janvier, février et mars 1985. 
physique nordique de Stakhanov, le barrage de Sanmen est inspiré des glorieux exemples ukrainiens, et les égouts de la ville de Luoyang sont plus grands que ceux de Léningrad. Cependant, durant le processus d'imitation, des «pertes" se produisent. Car, pour appliquer un modèle à la fois étranger et connoté comme tel, il faut en permanence composer avec la réalité. Durant son application, le modèle subit donc des modifications effectives qui, par principe, ne deviennent jamais explicites. Ces modifications varient. Il arrive, par exemple, que l'instrumentalisation du modèle produise, dans les conditions chinoises, un retour "intégriste " aux sources du modèle soviétique : par exemple, les camps de travail créés sur les normes soviétiques ont abrité un effort beaucoup plus réel qu'en URSS pour "rééduquer " idéologiquement les prisonniers ". Mais, le plus souvent, la pratique de l'instrumentalisation conduit au métissage ou à l'érosion du modèle. Ainsi, toujours dans le domaine pénal, l'entreprise de codification induite par le modèle soviétique s'est heurtée au poids des pratiques héritées du passé et aux hésitations de la direction chinoise sur le rôle du droit : la rédaction des codes, commencée très tôt, n'a été achevée qu'au début des années quatre-vingts. Et, dans la pratique, le lourd appareil judiciaire officiellement constitué au début des années cinquante a longtemps fonctionné de façon très allégée ${ }^{2}$.

Ces modifications ne sont pas innocentes. Elles préparent d'éventuelles décisions politiques sur le modèle. On a vu des régimes politiques dans lesquels l'inadaptation avérée du modèle soviétique inspirait son abandon partiel ou total. En Chine, les difficultés de l'instrumentalisation ont été largement compensées par les succès remportés. Néanmoins, elles ont suggéré certaines des transformations au moins verbales apportées par Mao Tse-tung aux institutions et à la politique économique inspirées par Moscou. Ainsi, le lourd appareil politico-administratif mis en place dans les années cinquante a été estimé impropre à la nécessaire mobilisation politique par le Chef. De même, la propagande industrialiste a été remplacée par un discours ruraliste.

Cependant, le cas chinois montre surtout le rôle des décisions politiques dans la trajectoire intérieure d'un modèle exogène. Jusqu'en 1958, l'histoire du modèle soviétique en Chine était surtout celle d'une greffe réussie - trop bien réussie, même, aux yeux de Mao Tse-tung. Car c'est lui qui a forcé la décision de recomposer ce modèle jugé dégénérescent. Par la suite, cet effort de recomposition a rapidement montré ses conséquences néfastes, mais il a fallu une nouvelle décision politique, issue d'une nouvelle lutte de factions, pour que l'usage du modèle soviétique en Chine soit à nouveau modifié.

La solution mixte qui a été adoptée à partir de 1978 paraît cependant de nature partiellement différente. Ce qui est en cause, ce n'est pas seulement le contenu de cette solution, c'est la façon dont elle a été adoptée. Autant la décision d'ouvrir le champ modernisateur au modèle

1. Pasqualini (Jean), Prisonnier de Mao, Paris, Gallimard, 1973.

2. Cohen (Jerome Alan), The criminal process in the PRC, 1949-1963. An introduction, Cambridge, Harvard University Press, 1968. 
occidental apparaît comme une décision volontariste des dirigeants, autant le recours instrumental au modèle soviétique d'organisation politique paraît spontané. Tout se passe comme si, après l'intermède maö̈ste, il était remonté à la surface par capillarité, à la suite d'un processus qui n'a trouvé que plus tard sa rationalité explicite.

Ce phénomène s'explique en partie par des facteurs historiques. Le fait, tout d'abord, que trois décennies de domination totalitaire avaient éliminé les représentants de l'idéologie libérale. Ou le fait que l'épuration maö̈ste a été idéologiquement plus ambitieuse mais au total infiniment moins meurtrière que les purges staliniennes : la majorité des "renégats » étaient encore vivants au moment de la succession... Mais le facteur décisif paraît avoir été la nature de l'expérience acquise dans les années cinquante. Encore embellie par le souvenir, cette expérience du modèle soviétique avait été triplement satisfaisante. Elle s'était tout d'abord révélée satisfaisante pour l'encadrement comme groupe d'intérêt : c'est alors que s'était constitué son pouvoir, c'est ensuite que ses avantages étaient devenus instables et dangereux. En second lieu, elle s'était avérée satisfaisante pour les administrateurs : après tout, la fidélité au dogme n'avait pas empêché accommodements concrets et nuances pratiques. En troisième lieu, l'application du modèle soviétique n'avait pas, par ellemême, induit de dépendance décisive vis-à-vis de l'extérieur. Ce ne sont pas les conseillers soviétiques, c'est l'alliance avec Moscou qui avait causé de graves difficultés. Enfin et surtout, cette période était dans l'ensemble une période d'ordre et d'unité, et le modèle soviétique, le seul modèle qui permît la survie du pouvoir communiste...

En d'autres termes, l'autonomie de décision sur le modèle dont disposent les dirigeants politiques comporte une limite, celle que constituent l'histoire, la psychologie et la sociologie de ces élites dirigeantes. Depuis 1949, en Chine populaire, elles ont changé d'avis sur à peu près tous les sujets importants. Sauf sur un point décisif, cependant : le maintien de leur propre monopole politique. De là un phénomène que les "pékinologues » connaissent bien : le vieillissement de ce milieu et la transmission du pouvoir dans son sein. Mais le plus intéressant, ce sont les conséquences de ces phénomènes. Car le vieillissement de l'élite favorise la constitution d'une mémoire collective qui est le résidu spontané de l'expérience. De chacune des tribulations du communisme chinois il reste ainsi une strate dans cette mémoire. Certaines strates sont recouvertes par d'autres, et certaines subsistent inentamées. Force est de constater que les méthodes politiques des années cinquante n'ont pas été toutes oubliées.

\section{LE MODÈlE SOVIÉTIQUE VU DE PÉKIN}

Finalement, la trajectoire historique du modèle soviétique en Chine ouvre un point de vue sur le modèle lui-même. Il n'existe qu'au travers de perceptions locales. De là d'intéressantes différences de perspective. Ainsi, alors que dans les pays occidentaux l'URSS apparaît d'abord 
comme «à l'Est », elle a toujours été perçue en Chine à la fois comme « à l'Est » et comme « au Nord »; et même, depuis 1949, sa dimension nordique s'est révélée plus stable et plus solide que sa dimension orientale. La position orientale du modèle soviétique a été contestée à Pékin de 1956 à 1978. Dès l'époque stalinienne, elle était compensée dans l'esprit des dirigeants de Pékin par sa rationalité nordique - vu souvent en Occident comme le « mangeur d'hommes", Staline était d'abord perçu dans le PCC comme le chef d'une puissante machine de pouvoir. Et, depuis 1978, la dimension « orientale " du modèle soviétique n'est admise qu'implicitement et comme intégrée dans la perception de l'URSS comme une super-puissance. En fait, la situation de la Chine « au Sud» et les tribulations de sa politique intérieure ont beaucoup influé sur la perception du modèle soviétique, au point, souvent, d'engendrer des erreurs de jugement. Ainsi, Pékin a successivement surévalué l'ampleur du « révisonnisme " khrouchtchévien, puis l'incapacité du régime de Brejnev à sortir d'une logique impérialiste étroite, et l'on assiste seulement depuis quelques années à un effort de Pékin pour construire une analyse plus factuelle du régime soviétique.

Outre son caractère largement nordique, le deuxième élément stable du modèle venu d'URSS est tout simplement qu'il est soviétique. Ce qui a convaincu les dirigeants du PCC d'y faire appel, ce n'est pas seulement son adaptation à leurs besoins, c'est aussi leur perception de la " réussite » du «pouvoir des soviets». A l'inverse, l'échec de la recomposition était aussi l'échec d'une substitution de la Chine à l'URSS, c'est-à-dire d'un pays du Sud à un pays du Nord, comme pôle mondial. Cet échec possède une raison de fond: le sens ne peut être totalement découplé de la puissance, contrairement à ce que Mao avait espéré. S'il est vrai qu'un modèle idéologique doit être porteur de sens, l'une de ses armes majeures est l'exemplarité ; il ne peut donc être véhiculé que par l'une des deux grandes puissances qui co-produisent le temps mondial. La stabilité, le renforcement même de la puissance soviétique depuis la fin des années cinquante transparaissent à travers l'échec de la recomposition maoiste.

Le paradoxe simplement apparent est que, si la recomposition d'un modèle idéologique paraît impossible à un grand pays du Sud, l'instrumentalisation, en revanche, s'avère à la fois aisée et relativement efficace. Non seulement les années 1949-1959 ont été les meilleures de l'histoire chinoise jusqu'en 1978, et cela dans tous les domaines à la fois, mais la greffe s'est avérée durable dans le domaine de l'organisation politique. Dans ce domaine au moins, le modèle soviétique s'est montré capable de résister aux mutations considérables survenues en Chine et dans le monde : il est aujourd'hui appliqué comme modèle d'organisation d'un pouvoir qui régit la modernisation. Ce résultat est d'une extrême importance car, pour être légitime, le monopole politique doit prétendre à plus que lui-même ; il s'enracine dans une prétention maintenue à la vérité, et donc à la reprise ultérieure d'une entreprise de totalisation temporairement interrompue. 
Il reste que le champ d'application de ce modèle a été réduit et que la modernisation elle-même lui est soustraite. L'URSS stalinienne était perçue, il y a quarante ans, comme un rival complet de l'Amérique capitaliste. Force est de reconnaître que, sur ce point, il existe un doute grave dans les milieux dirigeants chinois. La majorité estime probablement que l'arrivée au pouvoir de Gorbatchev constitue la dernière chance de mise à niveau de la puissance économique soviétique. Mais. pour certains, la cause est déjà entendue. Le modèle économique qui l'a emporté est le modèle occidental et la question posée est seulement celle du temps qu'il faudra pour que le différentiel de dynamisme économique produise un véritable déclin politique de l'URSS.

Au total, la problématique des relations cardinales paraît revêtir, dans l'analyse du cas chinois, un triple intérêt que d'autres travaux devront préciser. Tout d'abord, l'idée d'une polarisation cardinale de l'espacetemps mondial permet de mieux saisir cet espace comme contrainte et comme ressource, comme intérieur et extérieur, et donc de comprendre plus clairement les grands choix historiques des élites nationalistes chinoises. En second lieu, elle restitue, non seulement comme des intentions mais comme des réalités inscrites dans la durée, le travail des élites nationalistes sur les modèles exogènes. Travail évolutif, comme le montre le destin du modèle soviétique en Chine. Travail incertain également, comme l'indique l'évolution ambiguë des années récentes. Au total - c'est sans doute son intérêt principal - cette problématique met l'accent sur les rapports entre les phénomènes idéologiques et politiques dans l'espace et dans le temps. Son objet, certes, n'est pas d'expliquer leurs mécanismes endogènes. Mais elle permet de mieux percevoir leur déconnexion ou leur reconnexion, les sorties du temps et les resynchronisations. En ce sens, elle peut revêtir un grand intérêt pour les relations internationales comme discipline en facilitant leur rapprochement avec les autres disciplines qui rendent compte de l'évolution des formations sociales. 


\section{RÉSUMÉ DE L'ARTICLE/ABSTRACT}

\section{LA CHINE DANS L'ESPACE CARDINAL}

JEAN-LUC DOMENACH

Le premier paradoxe du communisme en Chine est que, si sa victoire est sortie d'une assomption de l'espace cardinal comme espace de contraintes et d'options, il a protégé son pouvoir d'une clôture totalitaire dont le modèle soviétique a été l'instrument et la limite. Un deuxième paradoxe est que l'exaltation de cette clôture engendrée par l'effort de recomposition du modèle soviétique a provoqué, après l'échec, le retour de la Chine dans l'espace cardinal et la «cardinalisation » de son espace intérieur. Cette trajectoire historique met en évidence le rôle du temps mondial comme temps des différentiels de dynamismes, et de l'espace mondial comme marché des valeurs en évolution. Mais la cotation de ces valeurs s'effectue à l'intérieur, les élites dirigeantes réalisent un travail sur les modèles exogènes, et l'espace politique subit les effets de ce travail.

\section{CHINA IN CARDINAL SPACE}

\section{JEAN-LUC DOMENACH}

One paradox of Chinese communism is that while its victory emerged from an assumption of cardinal space as a space of constraints and options, it protected its power by a totalitarian fence, the Soviet model being both the instrument and the limit. A second paradox is that the exaltation of this fence, brought about by the effort at recomposition of the Soviet model caused, after the failure, China's return to cardinal space and the "cardinalization" of its domestic space. This historical itinerary points out the role of world time as the time of differences in dynamism, and of world space as the market place of changing values. But the quotation of these values takes place inside; the ruling elites work on exogenous models, and political space registers the effects of that work. 